\title{
Optimal Classifier Based Spectrum Sensing in Cognitive Radio Wireless Systems
}

\author{
Siddharth Sharma \\ Department of Electrical Engineering \\ Indian Institute of Technology Kanpur \\ Kanpur - 208016, India \\ +91-9997773460 \\ sharmas@iitk.ac.in
}

\author{
Aditya K. Jagannatham \\ Department of Electrical Engineering \\ Indian Institute of Technology Kanpur \\ Kanpur - 208016, India \\ +91-512-2597494 \\ adityaj@iitk.ac.in
}

\begin{abstract}
In this work, we present and investigate the performance of novel classification schemes for spectrum sensing in cooperative multiple-input multiple-output (MIMO) wireless cognitive radio (CR) networks. In this context, we consider several optimal classification schemes such as support vector classifiers (SVC), logistic regression (LR) and quadratic discrimination (QD) for primary user detection. It is demonstrated that these classification techniques have a significantly reduced complexity of implementation in practical $\mathrm{CR}$ applications compared to conventional likelihood based detection schemes as they do not require knowledge of the channel state information and noise power. Further, in the presence of disruptive malicious users, the proposed classifiers have a significantly lower detection error compared to conventional detection schemes. Also, we propose a novel QD classifier for blind MIMO spectrum sensing scenarios. The detection performance of the proposed classifiers is compared with existing schemes in co-operative $\mathrm{CR}$ scenarios. It is demonstrated through simulation of several scenarios including the presence of malicious users, Doppler shift, and carrier frequency offset that the proposed classifiers offer a robust and significantly superior alternative to existing schemes for cooperative MIMO CR spectrum sensing.
\end{abstract}

\section{Categories and Subject Descriptors}

C.2.1 [Computer-Communication Networks]: Network Architecture and Design - Wireless Communication;

C.4 [Performance of systems]: Fault tolerance.

\section{General Terms}

Algorithms, Theory, Reliability.

\section{Keywords}

Cognitive Radio; Cooperative Spectrum Sensing; Support Vector Classifiers; Logistic Regression; Quadratic Discrimination.

\section{INTRODUCTION}

Cognitive radio (CR) networks have been proposed as a revolutionary breakthrough to enhance spectrum utilization through dynamic spectrum sharing. Conventional wireless networks are based on static allocation of spectral bands to a designated set of users, termed as the primary users. As demonstrated by the Federal Communications Commission (FCC) [1], and several other agencies, such a fixed assignment results in severe underutilization and scarcity of available spectrum. Hence, CR networks, in which a designated set of secondary users are empowered to opportunistically access unused spectrum bands for communication in the absence of the primary users, result in a significant enhancement of efficiency of the spectral utilization. A cognitive radio is defined as a wireless radio communication system that senses its electromagnetic environment and dynamically accesses the vacant spectrum bands, simultaneously adapting its operating parameters such as power, modulation order, coding rate etc. towards throughput maximization, while operating under the interference constraints mandated by the regulating authorities [2]. For instance, the CR based IEEE 802.22 standard for Wireless Regional Area Networks (WRAN), which utilizes white spaces in analog television bands, stipulates that the secondary users vacate the spectrum within two seconds of detection of the primary user.

In CR networks, for the secondary users to avoid interfering with the transmission of the primary users, it is critical to accurately sense the presence of concurrent transmission of the primary user in the spectrum band under consideration. Further, errors in the detection of the absence of the primary users lead to wasted spectrum opportunities for the secondary users. This key process of sensing the presence or absence of primary users is termed as spectrum sensing in the context of $\mathrm{CR}$ wireless networks. In a typical wireless communication scenario, thermal noise, shadowing and multipath fading significantly degrade the performance of spectrum sensing at any given isolated $\mathrm{CR}$ user. Hence, cooperative spectrum sensing, in which multiple CR users collaborate towards detection of primary user has been shown to be resilient to the above adversities of the wireless communication environment [3], [4].

In this context, we propose a support vector machine based classifier (SVC) and logistic regression (LR) based linear classifier for cooperative primary user detection in multiple-input multiple-output (MIMO) wireless CR networks. The proposed detection schemes have considerably lower complexity compared to the existing maximum-likelihood (ML) and maximum aposteriori (MAP) approaches for primary user detection since they do not require the knowledge of channel state information, which is significantly challenging in a cooperative MIMO wireless network as the complexity of channel estimation increases with the number of receive, transmit antennas and cooperating nodes. The proposed schemes do not require estimation of the noise power spectral density at the receiver, thus resulting in substantially simplistic detectors compared to existing schemes. Further, since the proposed classifiers are not based on 
the communicated channel state and noise PSD parameter estimates, their performance is highly robust compared to existing schemes in the presence of voluntary and involuntary report distortion arising due to malicious users and Doppler induced shifts respectively. Moreover, unlike existing SVM approaches in works such as [5], which employ classifiers based on non-linear kernel functions, the proposed classifiers have much lower complexity since they are linear. However, they do not result in a degradation of accuracy of spectrum sensing since it has been established in literature that linear classification is optimal in such matched filtering based additive white Gaussian noise baseband communication scenarios. Thus, intelligent integration of knowledge of the wireless communication environment with modern structural risk minimization based classifiers leads to the development of efficient low complexity approaches for spectrum sensing without compromising on the accuracy of the detection.

Further, we propose a novel quadratic discrimination (QD) based classifier for spectrum sensing in blind scenarios, where the transmitted symbols of the primary user are unknown. Moreover, the QD based classifier has a superior performance compared to conventional classifiers in the presence of non-idealities in the channel state information such as presence of carrier frequency offset, etc. Finally, we demonstrate the performance of the proposed classification schemes in the context of cooperative MIMO spectrum sensing through simulations. The rest of the paper is organized as follows. Section 2 describes the cooperative sensing system model. In section 3 we present the proposed SVM, LR, and QD techniques for spectrum sensing. Simulation results are presented in section 4, followed by conclusions in section 5 .

\section{SYSTEM MODEL}

Consider a cooperating spectrum sensing scenario comprising of $K \geq 1$ secondary CR users. Each CR user employs a multipleinput multiple-output (MIMO) wireless communication system with $N_{r}=N_{t}=M \geq 1$ antennas. Each secondary user (SU) senses the spectral band under consideration. Hence, the baseband system model at each SU can be described as,

$$
\boldsymbol{s}^{j}(\boldsymbol{n})=\boldsymbol{H}^{j} \boldsymbol{x}(\boldsymbol{n})+\boldsymbol{w}^{j}(\boldsymbol{n}),
$$

where $\boldsymbol{s}^{\boldsymbol{j}}(n) \in \mathbb{C}^{M}, \boldsymbol{w}^{\boldsymbol{j}}(n) \in \mathbb{C}^{M}$ are $m$-dimensional received signal and additive white Gaussian noise (AWGN) vector with covariance $\sigma_{n}^{2} \mathbf{I}$ respectively at the $j^{\text {th }}$ secondary user in the $n^{\text {th }}$ time instant. The quantity $x(\boldsymbol{n})$ denotes the transmit symbol vector. The matrix $\boldsymbol{H}^{\boldsymbol{j}} \in \mathbb{C}^{\boldsymbol{M} \times \boldsymbol{M}}$ denotes the flat fading MIMO channel matrix between the primary user and $j^{\text {th }}$ secondary user. The absence of a pilot beacon indicates availability of the spectrum band for communication of the CR users. Hence, the paradigm of spectrum sensing towards primary user detection reduces to a binary hypothesis testing problem, where the null hypotheses $\mathcal{H}_{0}$ and the alternative hypotheses $\mathcal{H}_{1}$ correspond to the absence and presence of the primary user respectively. Hence the competing hypotheses $\mathcal{H}_{0}$ and $\mathcal{H}_{1}$ can be formulated as,

$$
\begin{aligned}
& \mathcal{H}_{0}: \boldsymbol{s}^{\boldsymbol{j}}(n)=\boldsymbol{w}^{\boldsymbol{j}}(n) \\
& \mathcal{H}_{1}: \boldsymbol{s}^{\boldsymbol{j}}(n)=\boldsymbol{H}^{\boldsymbol{j}} \boldsymbol{p}(n)+\boldsymbol{w}^{\boldsymbol{j}}(n)
\end{aligned}
$$

The quantity $\boldsymbol{p}(n)=\mathbf{1}_{\boldsymbol{M}}=[1,1, \ldots, 1]^{T} \in \mathbb{R}^{\boldsymbol{M} \times \mathbf{1}}$ is the beacon pilot symbol transmitted by the primary user conveying its intention to actively use the corresponding spectrum band. As described earlier, the accuracy of primary user detection can be significantly enhanced and made robust through cooperation among the $\mathrm{CR}$ nodes. Hence, each $\mathrm{CR}$ user $1 \leq j \leq$ $K$ subsequently transmits the sensing measurements $\boldsymbol{s}^{j}(n)$ to a central data fusion center for further processing followed by decision mapping. This decision is then broadcasted back to the secondary users. Hence, the equivalent hypothesis testing at the fusion center can be formulated as,

$$
\begin{aligned}
& \mathcal{H}_{0}: \boldsymbol{s}(n)=\boldsymbol{w}(n) \\
& \mathcal{H}_{1}: \boldsymbol{s}(n)=\mathbb{H} \boldsymbol{p}(n)+\boldsymbol{w}(n)
\end{aligned}
$$

where $\boldsymbol{s}(n) \in \mathbb{C}^{K M}$ is the aggregated observation vector at the fusion center corresponding to stacking the individual $\mathrm{CR}$ observation vectors as,

$$
s(n)=\left[\left(s^{1}(n)\right)^{T},\left(s^{2}(n)\right)^{T}, \cdots,\left(s^{K}(n)\right)^{T}\right]^{T}
$$

and the corresponding concatenated channel matrix $\mathbb{H} \in \mathbb{C}^{K M \times M}$ is given as,

$$
\mathbb{H}=\left[\left(H^{1}\right)^{T},\left(H^{2}\right)^{T}, \cdots,\left(H^{K}\right)^{T}\right]^{T}
$$

The $2 M K$ dimensional vector, $\overline{\boldsymbol{s}}(n)=\left[\operatorname{Re}\left\{(\boldsymbol{s}(n))^{\boldsymbol{T}}\right\}, \operatorname{Im}\left\{(\boldsymbol{s}(n))^{\boldsymbol{T}}\right\}\right]^{\boldsymbol{T}}$, is formed by column wise stacking of the real and imaginary parts of the signal received at each of the $M$ antennas of $K$ secondary users. It can be readily seen from the above system model that the accuracy of detection using the conventional ML and MAP detection schemes depends critically on the knowledge of this aggregated channel matrix $\mathbb{H}$ and the noise variance parameter $\sigma_{n}^{2}$ [6]. However, accurate estimation of $\mathbb{H}$ necessitates a significant increase in the computational complexity at the SU. Further, the complexity increases considerably as the number of antennas $M$ increases. Hence, optimal classification techniques such as support vector machines (SVM), logistic regression (LR) and quadratic discrimination (QD), which do not require estimation of the channel coefficients and the knowledge of the noise variance, are ideally suited for such detection scenarios. In the next section, we provide an elaborate discussion of such classification techniques in the context of cooperative MIMO spectrum sensing.

\section{SPECTRUM SENSING TECHNIQUES}

The spectrum sensing schedule in cooperative $\mathrm{CR}$ scenarios comprises of an initial training phase consisting of $2 N_{b}$ training samples. Without loss of generality, the initial $N_{b}$ transmissions corresponding to transmission of the primary user beacon pilot symbols, followed by $N_{b}$ time instants i.e. $n=N_{b}+1, \ldots, 2 N_{b}$, corresponding to absence of the beacon. Hence, these $2 N_{b}$ vectors can be viewed as clusters of points in a $2 M K$ dimensional vector space occupying half spaces corresponding to the presence and absence of the primary users. Further, it is well known from results in the literature on statistical hypothesis testing that linear discrimination is optimal for detection in AWGN scenarios [7]. Below we describe the support vector machine and logistic regression based hyperplane construction for classifier based primary user detection.

\subsection{Support Vector Classifier}

The SVC aims to derive the optimal classifying hyperplane $\boldsymbol{a}^{\boldsymbol{T}} \overline{\boldsymbol{s}}(i)+b$ characterized by the normal vector $\boldsymbol{a}$, and distance parameter $b$ such that

$$
\begin{aligned}
& \boldsymbol{a}^{\boldsymbol{T}} \overline{\boldsymbol{s}}(i)+b \geq \mathbf{1}, \quad i=1, \ldots \ldots, N_{b} \\
& \boldsymbol{a}^{\boldsymbol{T}} \overline{\boldsymbol{s}}(i)+b \leq-\mathbf{1}, i=N_{b}+1, \ldots \ldots, 2 N_{b}
\end{aligned}
$$

The above classifier is termed as a strict linear discriminator, which classifies the training samples into two distinct halfspaces. However, in low SNR scenarios, as is often in the case of wireless communication, such a strict discriminator is not guaranteed to 
exist. This occurs due to the presence of outlying classification points arising due to fading and noise factors. Hence, a relaxed version of above discriminator can be formulated by introducing the non-negative slack variables, $\boldsymbol{u}, \boldsymbol{v} \in \mathbb{R}^{\mathbf{2 M K}}$. Therefore, the relaxed constraint based SVC can be cast as:

$$
\text { minimize }\left(\mathbf{1}^{T} u+\mathbf{1}^{T} v\right)
$$

subject to:

$$
\begin{gathered}
\boldsymbol{a}^{\boldsymbol{T}} \overline{\boldsymbol{s}}(i)+b \geqslant \mathbf{1}-\boldsymbol{u}_{\boldsymbol{i}}, \quad i=1, \ldots \ldots, N_{b} \\
\boldsymbol{a}^{\boldsymbol{T}} \overline{\boldsymbol{s}}(i)+b \leqslant-\mathbf{1}+\boldsymbol{v}_{\boldsymbol{i}}, i=N_{b}+1, \ldots \ldots, 2 N_{b} \\
\boldsymbol{u} \geqslant 0 \\
\boldsymbol{v} \geqslant 0
\end{gathered}
$$

It can be readily seen that the above problem corresponds to a standard form linear program [8] and hence can be readily solved using convex solvers such as CVX [9]. The SVC thus yields an optimal hyperplane that maximally separates the observation classes corresponding to the presence and absence of the primary user. An example of such an SVC based discriminator is illustrated in Figure 1 corresponding to a single user. Further, the proposed algorithm has much lower computational complexity as it does not employ a non-linear kernel [5] as is employed in conventional SVC based approaches. Moreover, it results in optimal detection performance since, as discussed earlier, linear discrimination is optimal in such scenarios [7]. Hence, closer analysis of the system model under consideration leads to the development of substantially low complexity classifiers without net degradation in performance.

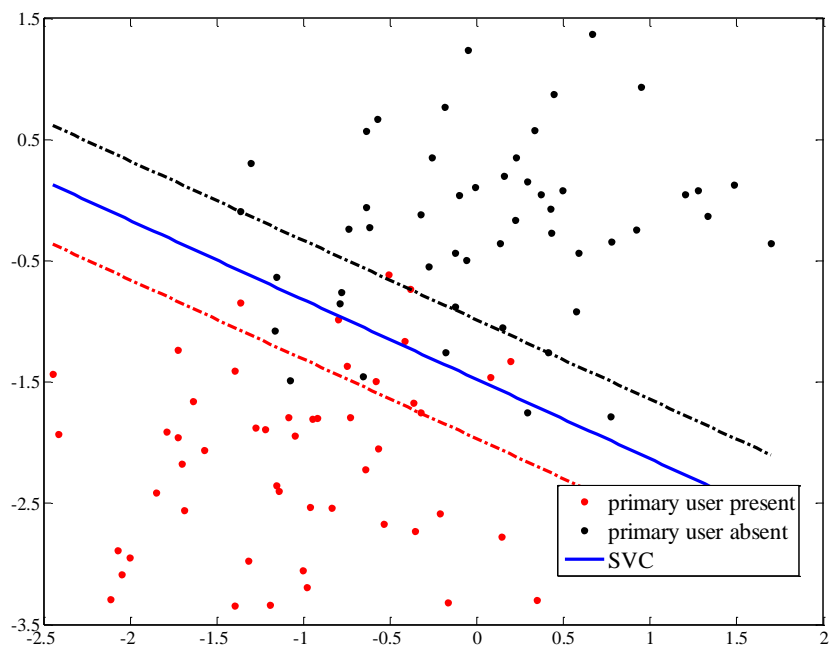

Figure 1. SVC based linear discrimination for spectrum sensing.

\subsection{Logistic Regression}

In this section, we describe a logistic regression based classifier for cooperative MIMO spectrum sensing. Let us define the binary random variable $y \in\{0,1\}$, as follows, $y=0,1$ denotes the absence and presence of the primary user respectively. The logistic regression based probability density function of $y$ can be described as,

$$
\begin{aligned}
& p_{0}=\operatorname{prob}(y=0)=\operatorname{prob}\left(H_{0}\right)=\frac{1}{1+e^{c^{T} \overline{\boldsymbol{s}}+d}} \\
& p_{1}=\operatorname{prob}(y=1)=\operatorname{prob}\left(H_{1}\right)=\frac{e^{c^{T} \overline{\boldsymbol{s}}+d}}{1+e^{c^{T} \overline{\boldsymbol{s}}+d}}
\end{aligned}
$$

The quantities $\boldsymbol{c}$ and $d$ which are the parameters of the LR, can be obtained by maximizing the log-likelihood function corresponding to training sets $\mathcal{T}_{0}=\left\{\overline{\boldsymbol{s}}(n) \mid \boldsymbol{x}(\boldsymbol{n})=\boldsymbol{p}(n), n=1,2, \ldots, N_{b}\right\}$ and $\mathcal{T}_{1}=\left\{\overline{\boldsymbol{s}}(n) \mid \boldsymbol{x}(n)=0, n=N_{b}+1,2, \ldots, 2 N_{b}\right\}$ as,

$$
\begin{aligned}
l(\boldsymbol{c}, d) & =\log \left(\prod_{i=1}^{N_{b}} \frac{e^{\boldsymbol{c}^{T} \overline{\boldsymbol{s}}(i)+\mathrm{d}}}{1+e^{\boldsymbol{c}^{T} \overline{\boldsymbol{s}}(i)+\mathrm{d}}} \prod_{i=N_{b}+1}^{2 N_{b}} \frac{1}{1+e^{\boldsymbol{c}^{T} \overline{\boldsymbol{s}}(i)+\mathrm{d}}}\right) \\
l(\boldsymbol{c}, d) & =\sum_{i=1}^{N_{b}}\left(\boldsymbol{c}^{\boldsymbol{T}} \overline{\boldsymbol{s}}(i)+\mathrm{d}\right)-\sum_{i=1}^{2 N_{b}} \log \left(1+e^{\boldsymbol{c}^{T} \overline{\boldsymbol{s}}(i)+d}\right)
\end{aligned}
$$

It can be seen that the above log-likelihood function is a concave function [5] of parameters $c$ and $d$. Hence, the ML estimate of $c, d$, corresponding to maximizing the log-likelihood function above can be formulated as a convex optimization problem. Figure 2 shows an example of the estimated logistical regressor for a randomly generated training set.

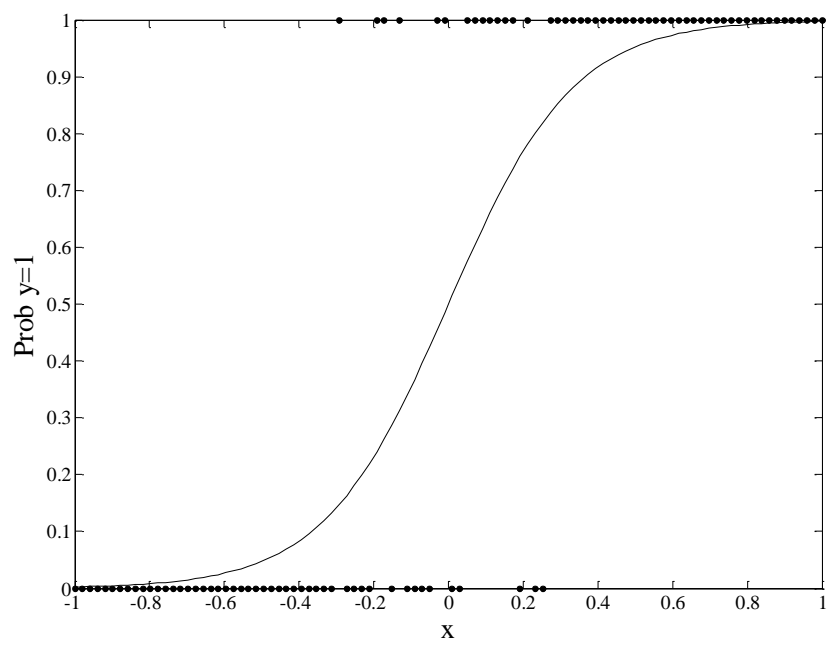

Figure 2. Logistic Regression

\subsection{Quadratic Discrimination}

The framework proposed above is not optimal for blind beacon vectors belonging to a given transmit symbol constellation. However, the above SVC can be readily modified as an energy kernel based QD classifier of the form:

$$
f(\boldsymbol{x})=\overline{\boldsymbol{s}}^{\boldsymbol{T}} \boldsymbol{P} \overline{\boldsymbol{s}}+\boldsymbol{q}^{\boldsymbol{T}} \overline{\boldsymbol{s}}+r,
$$

where $\boldsymbol{P} \in \mathbb{R}^{\mathbf{2} \boldsymbol{M} \boldsymbol{K} \times \mathbf{2} \boldsymbol{M} \boldsymbol{K}}$ is the positive semi-definite matrix, and $\boldsymbol{q} \in \mathbb{R}^{\mathbf{2 M K}}$ is $2 M K$ dimensional vector. This corresponds to separation of the hypothesis classes $\mathcal{H}_{0}, \mathcal{H}_{1}$ employing an ellipsoidal surface. Similar to SVC, since strict QD cannot often be guaranteed to exist in wireless training scenarios, this can be readily extended to a relaxed QD classifier. The QD characteristic parameters $\boldsymbol{P}, \boldsymbol{q}$, and $r$ can be computed as the solution of the convex optimization problem. Hence, the problem can be defined

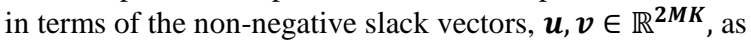

$$
\operatorname{minimize}\left(\mathbf{1}^{T} u+\mathbf{1}^{T} v\right)
$$

subject to:

$$
\begin{gathered}
\overline{\boldsymbol{s}}^{\boldsymbol{T}}(\boldsymbol{i}) \boldsymbol{P} \overline{\boldsymbol{s}}(i)+\boldsymbol{q}^{\boldsymbol{T}} \overline{\boldsymbol{s}}(i)+r \geqslant 1-\boldsymbol{u}_{\boldsymbol{i}}, \quad i=1, \ldots, N_{b} \\
\overline{\boldsymbol{s}}^{\boldsymbol{T}}(\boldsymbol{i}) \boldsymbol{P} \overline{\boldsymbol{s}}(i)+\boldsymbol{q}^{\boldsymbol{T}} \overline{\boldsymbol{s}}(i)+r \leqslant-1+\boldsymbol{v}_{i}, \quad i=N_{b}+1, \ldots, 2 N_{b} \\
\boldsymbol{P} \geqslant 0
\end{gathered}
$$






Figure 3. Signal constellation showing quadratic classifier and SVC's.

Figure 3 demonstrates the ellipsoid based quadratic classifier for a blind scenario where the transmit symbols of pilot beacon transmitted by the primary user are drawn randomly from the set $\sqrt{P_{d} / 2}( \pm 1 \pm j)$. Observe that two distinct linear SVC are required to achieve the performance identical to that of QD. Hence it results in reduction of computational complexity and is ideally suited for blind scenarios. Further, the QD classifier is robust to the distorting effects of carrier frequency offset arising at the receiver.

\section{SIMULATIONS AND DISCUSSION}

In this section we demonstrate the performance of the proposed SVC, LR and QD classifier schemes in the context of cooperative MIMO spectrum sensing for cognitive radio scenarios. We consider the total number of cooperative secondary users in the range $1 \leq K \leq 7$ and number of receive and transmit antennas of the MIMO system in the range $1 \leq M \leq 7$. We consider a training set of size $2 N_{b}=200$ training samples with $N_{b}=100$ each for the case of presence and absence of the primary user. The performance of the classifiers is evaluated over a set of $n_{v}=$ 1000 samples. We employ the standard metrics of probabilty of false alarm and probability of miss detection to characterize the performance of the proposed classification schemes. Further, the performance of the proposed techniques is compared with that of the conventional maximum likelihood detector (ML). The ML detector requires knowledge of the noise variance $\sigma_{n}^{2}$ and channel state information. The optimal ML decision rule which minimizes the probability of error for choosing one of the two competing hypothesis is given as [9],

$$
\begin{aligned}
& \mathcal{H}_{0}: d_{0}(n) \leq d_{1}(n) \\
& \mathcal{H}_{1}: d_{0}(n)>d_{1}(n)
\end{aligned}
$$

where $d_{0}(n)=\|\overline{\boldsymbol{s}}(n)\|, d_{1}(n)=\left\|\overline{\boldsymbol{s}}(n)-\sqrt{\rho} \mathbb{H} \mathbf{1}_{\boldsymbol{M}}\right\|$, and $\mathbf{1}_{\boldsymbol{M}}=$ $[1,1, \ldots, 1]^{T} \in \mathbb{R}^{\boldsymbol{M} \times \mathbf{1}}$. Finally the performance of the proposed schemes is also compared with that of the sub-optimal K-means clustering (KM) technique. The performance of the competing algorithms for varying number of secondary users and antennas are shown in Figure 4 and Figure 5 respectively. It can be seen that the classification error of the SVC and LR classifiers is comparable to that of the optimal ML detector. Thus, these classifiers are ideally suited for cooperative spectrum sensing since they do not compromise on detection performance while reducing the overhead of channel state information and noise power estimation. Further, it can be observed that the detection performance of all the schemes improves progressively with increasing number of cooperating secondary users and MIMO system antennas as expected.

A typical adversity for detection in wireless communication systems is the time selectivity of the underlying wireless channel. This occurs due to the Doppler shift arising from the mobile nature of wireless nodes. Such a time varying channel can be described by the first order auto regressive (AR) model,

$$
\boldsymbol{H}(n)=a \boldsymbol{H}(n-1)+\boldsymbol{V}(n),
$$

where $a$ is the AR coefficient and $\boldsymbol{V}(n)$ is the model noise. We simulate the performance of the proposed SVC scheme for cooperative spectrum sensing in this context of time varying MIMO channel for $K=5$ secondary users and different values of the AR model coefficient $a$. This is demonstrated in Figure 6. From this, it can be see that the classification performance of the proposed SVC scheme is resilient to the time variation of the channel state information and degrades only slightly with increasing Doppler shift. Hence, SVC and other such classification schemes are ideally suited for primary user detection in cooperative time varying MIMO scenarios.

Further, malicious users can subvert the spectrum sensing process at the fusion center by willful manipulation of the reported measurements. This leads to poor detection performance in the conventional ML detector since it cannot effectively distinguish between malicious and cooperative users. However, by employing a random training phase, in which the presence and absence of the beacon are switched randomly, the SVC and LR classification schemes can be made resilient to malicious user manipulation. This is due to the fact that such classifiers automatically assign lower weights to the information provided by the malicious user since they appear as outliers with correspondingly high slack values $\boldsymbol{u}_{\boldsymbol{i}}$ and $\mathbf{v}_{\boldsymbol{i}}$. Also, the value of the weight vector $\boldsymbol{a}$, corresponding to the malicious users is low. Figure 7 illustrates the performance of the SVC and LR techniques when the malicious user transmits random information during the training phase. It can be clearly seen that the detection performance of the SVC and LR scheme is significantly superior to that of the ML detector in presence of malicious user.

Similar to the conventional likelihood based approaches, the SVC and LR schemes also enable a tradeoff between the probability of false alarm and the probability of missed detection. This can be achieved by varying the distance parameter $+1,-1$. For instance, modifying these thresholds to $+1.5,-0.5$, results in decrease in probability of false alarm while raising the probability of missed detection. This effect of modifying the threshold in case of LR is demonstrated in Figure 8, where it can be seen that probability of missed detection decreases at the cost of increased probability of false alarm as the threshold is decreasing.

As discussed in section 3.3, the QD-based classifier is particularly resilient when the receiver has a carrier frequency offset. We model such a channel in the simulations as,

$$
\boldsymbol{H}_{\boldsymbol{i j}}(\boldsymbol{n})=\boldsymbol{H}_{\boldsymbol{i j}}(\mathbf{0}) e^{j n \theta}, \text { where } \theta=0.0015 .
$$

Figure 9 demonstrates the classification performance for this carrier frequency offset. 


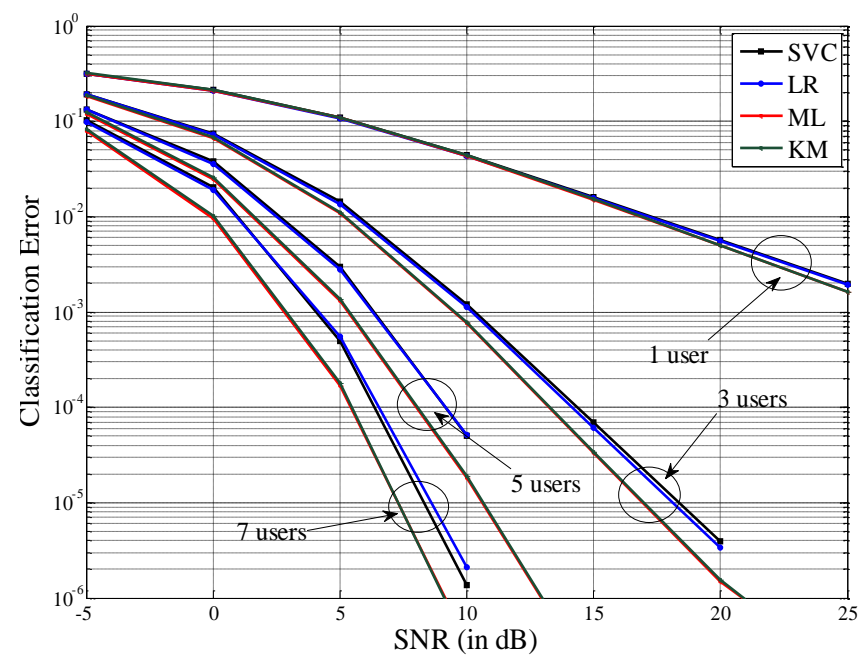

Figure 4. Classification error performance of SVC, LR, Kmeans clustering (KM) and Maximum Likelihood (ML) for varying number of users

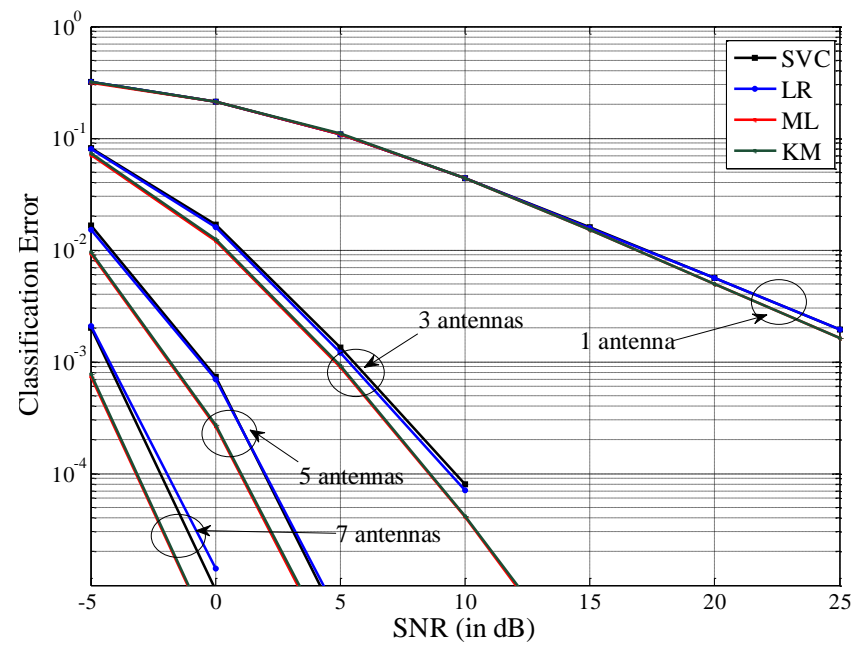

Figure 5. Classification error performance of SVC, LR, Kmeans clustering and Maximum Likelihood with varying number of antennas.

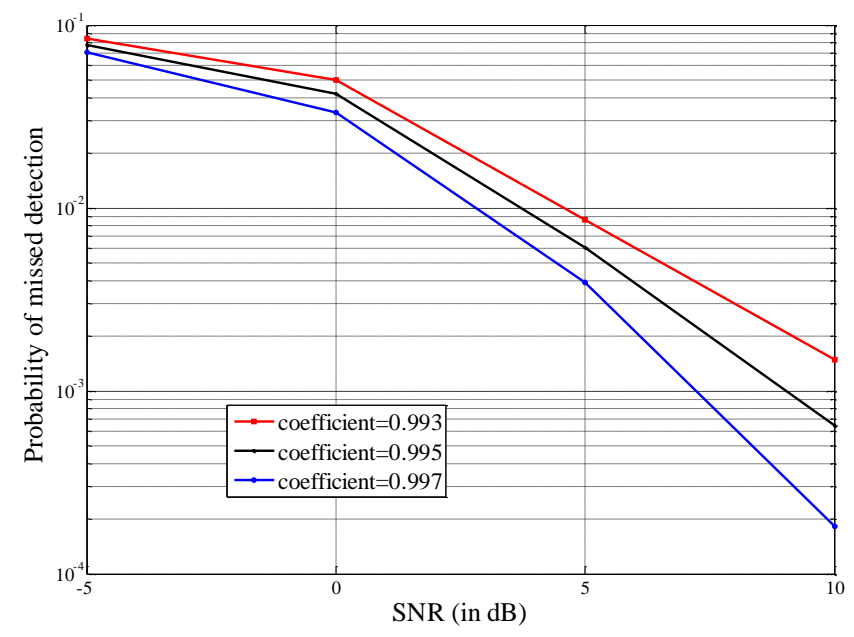

Figure 6. Probability of missed detection using SVC, time varying channel, $A R$ model, and $K=5$ secondary users.

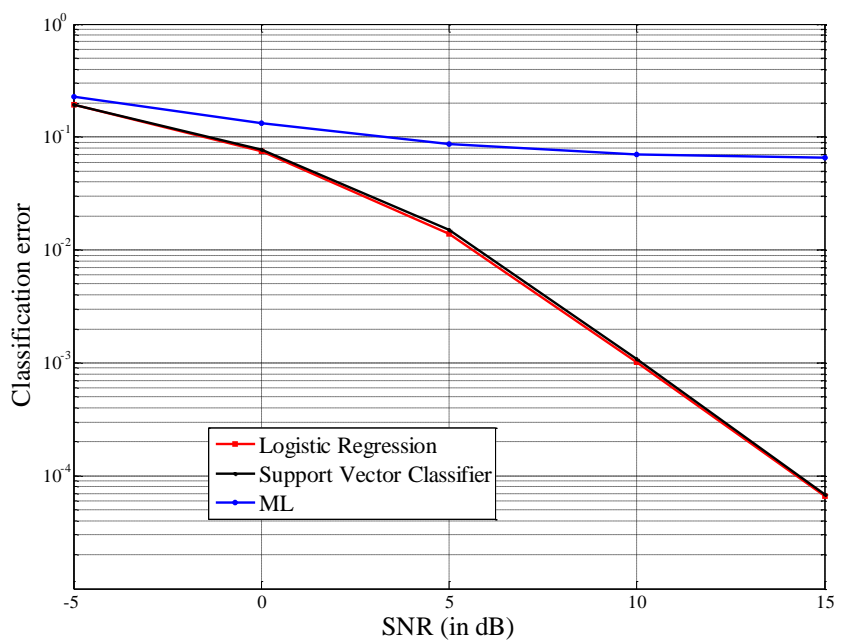

Figure 7. Classification error performance of SVC, LR and, ML detector, with $K=4$ secondary users including one malicious user.

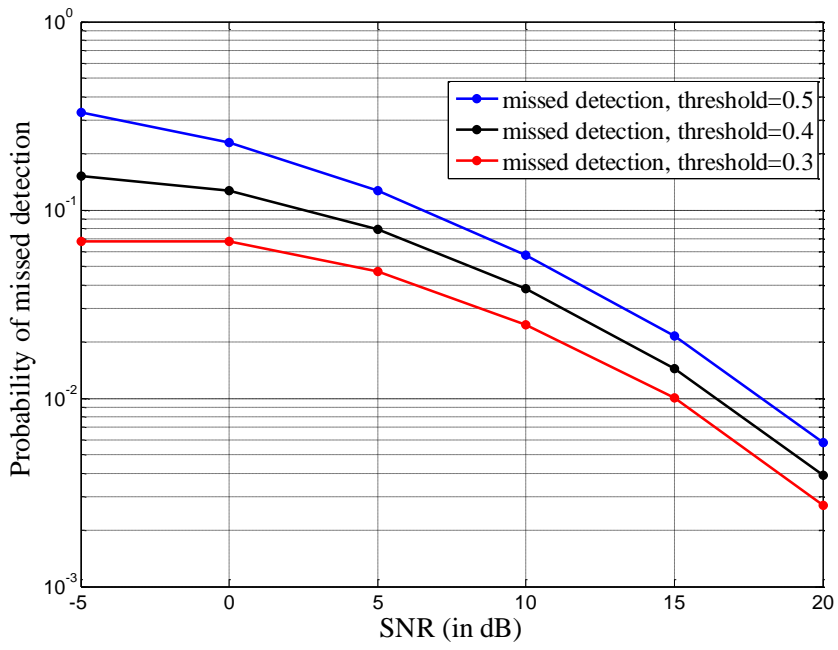

Figure 8. Probability of missed detection of LR technique with varying threshold

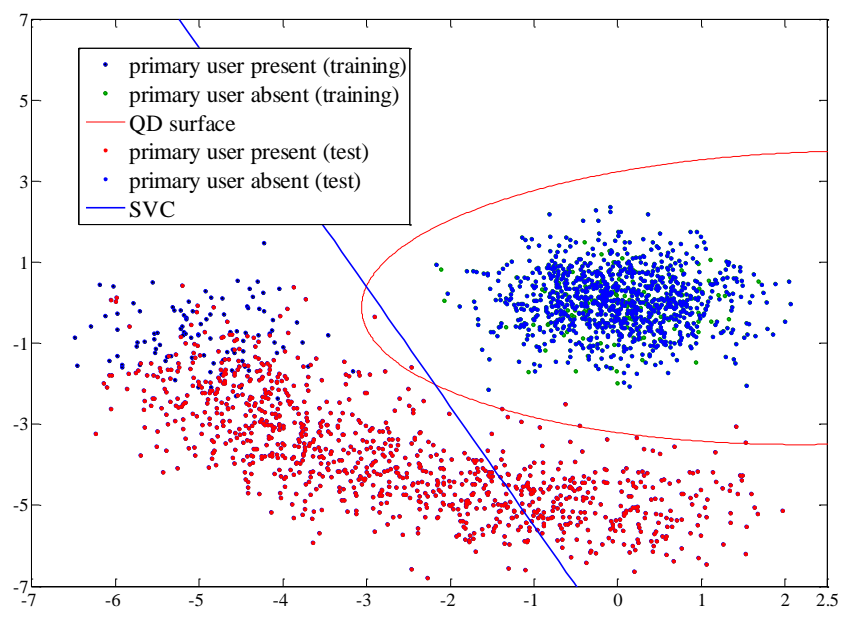

Figure 9. Signal constellation, showing the QD classifier and the SVC in case where the channel has a carrier frequency offset. 


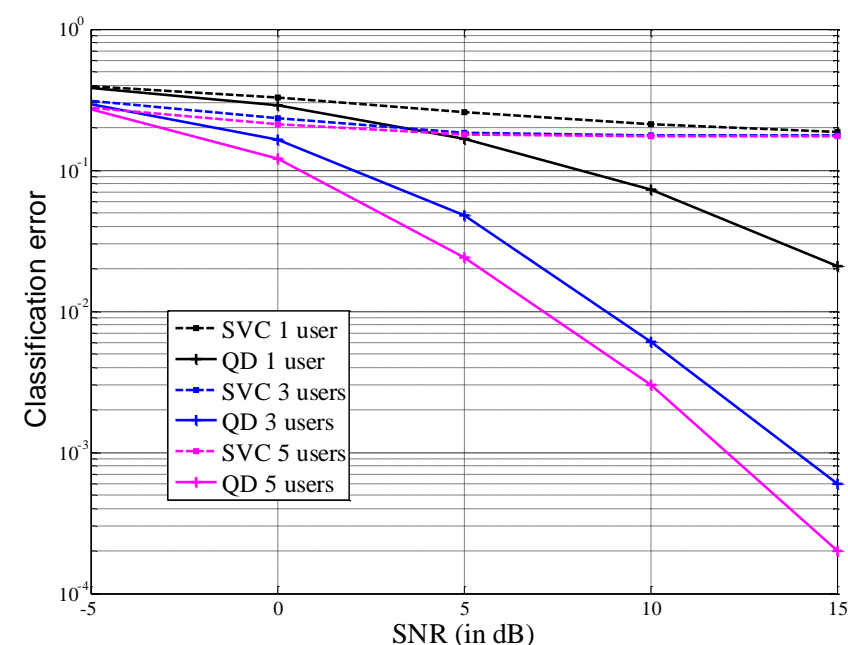

Figure 10. Classification error performance of SVC and QD in presence of carrier frequency offset.

It can be readily seen from the results in Figure 10, that the QD technique has superior performance when compared to SVC. Also, for varying number of secondary users, it can be observed that QD consistently performs better.

\section{CONCLUSIONS}

In this paper, we have demonstrated novel applications of the SVC, LR and QD classifiers in the context of cooperative MIMO spectrum sensing. The major advantage of the proposed schemes over conventional likelihood based detection is that they do not require the knowledge of the channel and noise variance parameters. Further, the SVC and LR schemes offer significant immunity to the distorting effects of channel fade, time selectivity and malicious users. It has also been shown that QD is ideally suited for spectrum sensing in blind scenarios and in the presence of a carrier frequency offset. Hence, the proposed SVC, LR and QD schemes provide a significant performance advantage in the context of cooperative MIMO spectrum sensing.

\section{ACKNOWLEDGMENTS}

Our thanks to ACM SIGCHI for allowing us to modify templates they had developed.

\section{REFERENCES}

[1] FCC, "Spectrum policy task force report," in Proceedings of the Federal Communications Commission (FCC '02), Washington, DC, USA, November 2002.

[2] FCC, "Notice of proposed rule making and order: Facilitating opportunities for flexible, efficient, and reliable spectrum use employing cognitive radio technologies," ET Docket No. 03-108, Feb. 2005.

[3] Unnikrishnan, J.; Veeravalli, V.V.; , "Cooperative Sensing for Primary Detection in Cognitive Radio," Selected Topics in Signal Processing, IEEE Journal of , vol.2, no.1, pp.1827, Feb. 2008

[4] Mishra, S.M.; Sahai, A.; Brodersen, R.W.; , "Cooperative Sensing among Cognitive Radios," Communications, 2006. ICC '06. IEEE International Conference on , vol.4, no., pp.1658-1663, June 2006.

[5] Luca Bixio, Marina Ottonello, Mirco Raffetto, and Carlo S. Regazzoni, "Comparison among Cognitive Radio Architectures for Spectrum Sensing," EURASIP Journal on Wireless Communications and Networking, vol. 2011.

[6] Yonghong Zeng, Ying-Chang Liang, Anh Tuan Hoang, and Rui Zhang, "A Review on Spectrum Sensing for Cognitive Radio: Challenges and Solutions," EURASIP Journal on Advances in Signal Processing, vol. 2010.

[7] Kay, SM. Fundamentals of Statistical Signal Processing: Detection Theory, Englewood Cliffs, N.J: Prentice-Hall PTR, 1993.

[8] S. Boyd, L. Vandenberghe. Convex Optimization. Cambridge University Press, 2004.

[9] M. Grant and S. Boyd. CVX: Matlab software for disciplined convex programming, version 1.21. http://cvxr.com/cvx, April 2011. 